\title{
Validación de la percepción de los conocimientos metodológicos de los estudiantes del Centro Universitario Regional San Pedro Sula de la Universidad Pedagógica Nacional Francisco Morazán
}

\section{Validation of the perception of the methodological knowledge of the students at the San Pedro Sula Regional University Center of the Francisco Morazán National Pedagogical University}

Pastor Umanzor ${ }^{l}$

\section{Resumen}

Con este estudio se validan las percepciones de los estudiantes del Centro Universitario Regional San Pedro Sula (CURSPS) de la Universidad Pedagógica Nacional Francisco Morazán (UPNFM) sobre sus conocimientos metodológicos en función de sus saberes sobre el modelo educativo de la UPNFM y sus componentes: planes de estudio, guión metodológico, planes de clase, recursos y medios, y formas de evaluación de sus aprendizajes. La validación se hace con una escala Likert con 57 afirmaciones y aplicada como encuesta a 91 estudiantes matriculados en Metodología de Investigación Cuantitativa y en Metodología de Investigación Cualitativa, II período, 2020. Los objetivos de la validación de la escala se cumplieron por encima de las exigencias estadísticas esperadas y por eso se muestra que los conocimientos metodológicos de los estudiantes son explicados por sus saberes sobre los planes de clases, los recursos y medios, los planes de estudio y las formas de evaluación de sus aprendizajes.

Palabras Claves: Conocimientos metodológicos, planes de clase, recursos y medios, planes de estudio,evaluación

1pumanzor@upnfm.edu.hn. Doctor en Educación. Centro Universitario Regional de San Pedro Sula, Universidad Pedagógica Nacional Francisco Morazán. Honduras. https://orcid.org/0000-0002-1620-6543 


\begin{abstract}
This study validates the perceptions of the students of the San Pedro Sula Regional University Center (CURSPS) of the Francisco Morazán National Pedagogical University (UPNFM) about their methodological knowledge based on their knowledge about the UPNFM educational model and its components: study plans, methodological script, lesson plans, learning resources, and forms of evaluation of their learning. The validation is done with a Likert scale with 57 statements and applied as a survey to 91 students enrolled in Quantitative Research Methodology and Qualitative Research Methodology, II period, 2020. The scale's validation objectives were met above the expected statistical demands and that is why it is shown that the methodological knowledge of the students is explained by their knowledge about the lesson plans, learning resources, the study plans and the forms of evaluation of their learning.
\end{abstract}

Keywords: Methodological knowledge, lesson plans, learning resources, study plans, evaluation

\title{
Introducción
}

El estudio se realizó a partir de reflexiones relacionadas con el desarrollo del modelo educativo, desde el 2014, por la UPNFM para formar los docentes de Honduras. Este modelo, teóricamente, se sustenta en el enfoque de formación basado en competencias y por eso se deben formar docentes competentes en la enseñanza, para fortalecer el aprendizaje de los estudiantes para saber enseñar.

Este modelo tiene como componentes: una fundamentación teórica y conceptual, planes de estudio (currículo), guión metodológico, procesos de planificación didáctica que siguen los profesores universitarios en la formación docente de un profesor, quienes dominan métodos, técnicas, estrategias y procesos de enseñanza propios de conocimientos metodológicos cimentados en la ciencia didáctica, así como de las formas de evaluación de los aprendizajes.

De aquí emanan las directrices para que los profesores fomenten en sus estudiantes una formación docente de un profesor con los conocimientos científicos y pedagógico-didácticos 
que resistan el proceso de enseñanza-aprendizaje venido de la ciencia didáctica y desde el cual se fortalecerá la formación de profesores competentes.

Esta tarea, en la universidad, pareciera que es responsabilidad solamente de los profesores, pues las capacitaciones alimentan la idea de ser críticos, reflexivos y creativos e innovadores, pero el centro de sus acciones didácticas se reduce al desarrollo de prácticas pedagógicas más convencionales que a la formación de criterios de la teoría crítica. Esto quizás se deba a que la ciencia didáctica es vista como una disciplina de la pedagogía en el modelo educativo. Luego, cuando de las formas de evaluación de los aprendizajes de los estudiantes se siguen utilizando los criterios conductistas de la evaluación, pues la evaluación de los aprendizajes se basa en objetivos y no competencias.

A esto hay que agregar que faltan precisiones epistemológicas que determinen el acervo cultural con el que debe egresar un profesor de la universidad (Lorenzo, 2017). El mismo modelo educativo se decanta por describir el constructivismo como el enfoque fundamental y deja al de la formación basada en competencias sin mayores explicaciones teóricometodológicas.

Si la formación basada en competencias implica la formación docente de un profesor competente, se debería desarrollar la teoría de Tobón (2015) que implica, primero, formar a la persona que será profesor (saber ser); segundo, formar el caudal científico y metodológico de los profesores para que respondan a las exigencias académicas competentes (saber conocer); y, tercero, formar las capacidades metodológicas en los profesores para un desempeño competente (saber hacer).

Hasta ahora, no se tienen evidencias estadísticas de que el modelo educativo con el enfoque de formación docente basado en competencias haya tenido éxito. Entre profesores se comenta que no están preparados para enseñar y evaluar en competencias (Umanzor y Castro, 2019). Entre estudiantes se dice que sus maestros se han orientado a impartir un contenido sin el apoyo didáctico concurrente (Rodríguez Rivera, López Molina, Arias Galindo, Mejía González y García Menjívar, 2020). 
De lo que se colige que la formación de los profesores en Honduras está constituida por la construcción de conocimientos científicos y pedagógicos que dispensan los formadores de formadores en sus cátedras. Cada formador de formadores muestra un discurso pedagógico construido en las aulas, como un profesor, pero que responde a una formación específica en una ciencia, como su profesión, no en la enseñanza de las ciencias como debería ser su nueva profesión en una universidad (Zabalza, 2009); cada profesor aprendió una ciencia y su método científico como su interés central, no el ser profesor. Quiere decir que la universidad debe intervenir para formar un profesor competente, bajo la tesis que, si forma profesores, lo debe hacer un profesor.

Cuando se habla de validar conocimientos metodológicos de los estudiantes universitarios es porque en el discurso de los profesores y estudiantes están presentes prácticas didácticas arraigadas con el uso de métodos, técnicas, estrategias y procesos de enseñanza que no están fundamentadas en la ciencia didáctica y eso debilita la formación docente de los profesores.

Esto puede hacer aparecer confusiones teórico-conceptuales que podrían estar asociadas con la pérdida del sentido de lo académico en las concepciones sobre la enseñanza, el aprendizaje y las prácticas pedagógicas de los profesores universitarios, pues como dice Díaz Barriga (2005):

nos enfrentamos a un momento social que ha generado la pérdida del sentido del trabajo académico por parte de los propios docentes de las instituciones educativas. Pérdida que se refleja en el descuido de los espacios académicos. La institución educativa contemporánea parece no conceder importancia al trabajo, discusión y diálogo entre su personal académico. (p. 44)

El mismo autor antes había dicho que "cuando la didáctica sólo se enseña como un saber técnico, las diferentes propuestas didácticas solamente se visualizan como diferentes posiciones técnicas, incluso como diferentes 'modas"”. (1986, p. 6) 
En el quehacer didáctico en las aulas hay un intercambio de conceptos didácticos que se imbrican y le dan sentido epistemológico y metodológico a la formación docente de un profesor, pues allí es donde un formador de formadores crea las condiciones que facilitan al estudiante ser profesor con los conocimientos metodológicos propios de un docente competente.

Estas distancias conceptuales y epistemológicas entre profesores y estudiantes son el objeto de la validación de los conocimientos metodológicos que manejan los estudiantes, en su proceso de formación como docentes, por eso se pregunta: ¿de qué manera los conocimientos metodológicos de los estudiantes del CURSPS-UPNFM son explicados por sus saberes sobre planes de clases, recursos y medios, planes de estudio y formas de evaluación de los aprendizajes definidos por el modelo educativo para la formación de docentes críticos, reflexivos y creativos?

El objetivo general era validar la percepción que los estudiantes poseen sobre sus conocimientos metodológicos en función de sus saberes del modelo educativo y sus componentes: planes de estudio, guión metodológico, planes de clases, recursos y medios, y las formas de evaluación de los aprendizajes propugnados por la universidad para la formación de docentes críticos, reflexivos y creativos.

Este objetivo se alcanzó con el logro de los objetivos específicos siguientes: 1. Identificar la situación socio-demográfica, de conectividad y laboral de los estudiantes para su formación como profesores; 2. Determinar los niveles de conocimientos metodológicos, del modelo educativo, de los planes de estudio, del guión metodológico, de los planes de clases, de los recursos y medios, y de las formas de evaluación de sus aprendizajes; 3. Establecer la relación existente entre los conocimientos metodológicos de los estudiantes y los del modelo educativo y sus componentes que se validan con la escala Likert; y, 4. Explicar cómo los conocimientos metodológicos de los estudiantes del CURSPS-UPNFM son determinados por sus saberes sobre planes de clases, recursos y medios, planes de estudio y las formas de evaluación de sus aprendizajes en la formación de profesores competentes. 


\section{Discusión Teórica}

El ser profesor implica tener claras concepciones sobre la enseñanza y desarrollar prácticas pedagógicas contextualizadas, condiciones que hacen ver a los profesores con un discurso pedagógico congruente, coherente, consistente y con unidad en un profesor que sabe enseñar desde el aprender (Umanzor, 2018).

Un discurso pedagógico construido en las ciencias de la educación y fortalecido con una filosofía de enseñanza propia y una pedagogía específica que lo perfila como profesor y que puede decidir el tipo de formación a desarrollar, con sólidos conocimientos metodológicos venidos del uso y manejo adecuados de métodos, técnicas, estrategias y procesos de enseñanza aprendidos en la ciencia didáctica que direccionan el proceso enseñanzaaprendizaje, con el conocimiento y manejo de las teorías del aprendizaje y de la conducta humana que facilitan el aprendizaje significativo en los estudiantes; $y$, con dominio de las teorías sociológicas que favorecen una comunicación eficaz con las masas en la formación de los profesores en las aulas (Bolívar, 2005).

Con estas expectativas, el profesor está compelido a aprender a enseñar para enseñar desde el aprender (Gallego y Pérez, 2003) considerando que su satisfacción como profesional de la enseñanza está centrada en el aprender a reconstruir sus aprendizajes en la enseñanza. Enseña quien aprende y de esa manera es capaz de fortalecer los conocimientos científicos de sus estudiantes impulsado por una actitud científica (Bourdieu, 2002) que lo respalda.

La formación docente de un profesor en la UPNFM está basada en sus fundamentos filosóficos, pedagógicos, didácticos, psicológicos, psicopedagógicos, metodológicos y sociológicos definidos en su modelo educativo, los planes de estudio y el enfoque de formación basado en competencias. La UPNFM (2014) aspira a la formación de un profesor que sea "crítico, reflexivo y creativo", competente, en otras palabras.

En este caso, los alcances del modelo educativo son considerados como el principal argumento teórico de esta investigación. Este modelo educativo es el que define el tipo de profesor a formar en competencias. De allí que es parte de la discusión teórica y se completa el marco teórico con aportes de otros investigadores sobre el tema. A continuación, 
se presenta un resumen de la teoría establecida en el modelo educativo y luego las teorías que apoyan la formación docente de un profesor.

\section{El modelo Educativo}

La UPNFM propugna la formación de docentes centrada en el desarrollo de las competencias científicas, humanísticas y tecnológicas innovadoras. El modelo es un patrón conceptual a través del cual se orienta el quehacer académico y administrativo para facilitar una perspectiva filosófica, psicológica y pedagógica, así como el logro de las metas institucionales y el cumplimiento de su misión.

La postura de la UPNFM en la formación de docentes en el país parte del logro de los siguientes objetivos: formar y perfeccionar los docentes del país; contribuir al desarrollo de la investigación científica; contribuir a la conservación e incremento del patrimonio cultural y natural del país; $y$, vincular sus actividades con entidades nacionales y extranjeras que persigan los mismos objetivos.

Para el logro de estos objetivos la universidad asume los siguientes principios y valores: responsabilidad; justicia; respeto y aceptación de los otros; equidad; transparencia; democracia; solidaridad; servicio de calidad; liderazgo en la educación; $y$, tolerancia con diálogo.

Con base en estos principios y valores se desarrollan las políticas institucionales centrados en: La formación de docentes es la función principal, la investigación es la actividad prioritaria para la construcción de conocimiento, y la extensión del conocimiento científico es lo que la vincula a la sociedad hondureña.

\section{Fundamentación del Modelo}

Para los propósitos de esta investigación solamente se consideran los fundamentos: filosófico, pedagógico, psicológico, psicopedagógico y metodológico. La fundamentación filosófica propugna por el desarrollo del constructivismo-humanista donde el estudiante debe aprender a pensar, buscar soluciones y tomar decisiones mediante la investigación activa y participativa. 
La fundamentación psicológica sugiere la coexistencia de diversos paradigmas: conductista, humanista, cognitivo, psicogenético y sociocultural, pero se asumen los aportes de Piaget (1952), Vygotsky (1978), Ausubel (1963) y Bruner (1960) como los que mejor se complementan con el objetivo de la formación basada en competencias, pues su interés es: identificar y construir condiciones de aprendizaje de vinculación de la teoría con la práctica; diseñar experiencias de aprendizaje para arribar a diferentes soluciones; crear entornos cooperativos, colaborativos y de apoyo; priorizar estrategias didácticas para descubrir y construir conocimiento por sí mismos; identificar niveles de desempeño para la evaluación; y, promover el método de resolución de problemas.

La fundamentación pedagógica resalta que los estudiantes construyan su propio conocimiento. Por eso las estrategias de aprendizaje, enseñanza y evaluación están sustentadas en el método didáctico constructivista y el método transdisciplinario de la mayéutica. Cabe destacar que la didáctica es una disciplina dentro de la pedagogía y en ese marco, la investigación profundizará la relación teoría-práctica en la formación docente; la extensión generará espacios para la construcción de nuevos conocimientos y fortalecerá la función social de la universidad para actualizar el currículo.

La fundamentación psicopedagógica aboga para que el estudiante construya su aprendizaje y que el docente propicie los ambientes de enseñanza con los que el primero pueda aprender a aprender. Y la fundamentación metodológica se orienta con los planes de estudio como la base de la formación de los docentes en una concepción de formación integral en competencias que lo hagan ser, saber y saber hacer, lineamientos tomados del Proyecto TUNING Latinoamérica adaptados al contexto hondureño, en función de las competencias: genéricas, específicas profesionales y disciplinares.

En esta fundamentación metodológica no hay referencias a los conocimientos metodológicos que debe poseer un estudiante ni los dominios asociados con métodos, técnicas, estrategias y procesos de enseñanza. Falta precisar cómo se fortalecerán las competencias genéricas, específicas profesionales y las disciplinares para formar un docente competente, así cómo se 
capacitará a los docentes universitarios para conducir la formación docente de un profesor competente. El modelo educativo acepta que las competencias docentes, investigativas y extensionistas son parte del acervo cultural de cada profesor universitario y eso garantiza la formación del tipo de docente competente.

\section{Elementos del Modelo}

Los elementos del modelo educativo son: el contexto, los componentes filosóficos, la concepción de hombre, docente, estudiante y comunidad educativa, los componentes organizacionales, $\mathrm{y}$, los componentes académicos que cimentan el tipo de persona a formar, los activos de la educación, los potenciadores de la formación docente, $\mathrm{y}$, la cultura hondureña.

De allí surgen los pilares de la formación de los docentes: Enseñanza personalizada que sugiere un aprendizaje autónomo basado en su propia iniciativa para resolver problemas. Lo que lleva a profesores y estudiantes a aprender por sus propias experiencias concretas en la docencia. Por tanto, se garantiza un aprendizaje creativo por la necesidad de cambio. Estos pilares garantizan una enseñanza personalizada tanto de sus profesores como de sus estudiantes, especialmente de aquellos que laboran como docentes y que quieren perfeccionar sus prácticas pedagógicas anteriores.

Los elementos del modelo están claros, pero no sus aplicaciones para la formación del tipo de docente que se debe formar. Hace falta una acción que direccione y produzca los efectos esperados en la formación del profesor perfilado.

\section{Implicaciones del Modelo Educativo}

Con el modelo por competencias se espera que las personas desarrollen actitudes de apertura, habilidades y el aprendizaje de contenidos de forma equilibrada. El modelo está centrado en desempeños relevantes en la enseñanza y el aprendizaje, conocimiento teórico, participación en investigaciones, para una acción transformadora.

La universidad asume que desde la investigación se planifica la acción social y mediante el desarrollo de una docencia inspirada en la investigación educativa se mejora el quehacer 
del profesorado: su perfeccionamiento y formación en función de las evidencias empíricas o teóricas validadas en la sociedad por el encuentro de la ciencia con la solución de los problemas de la comunidad.

En este caso, los alcances de los planes de estudio se limitan a las cartas descriptivas que son el soporte científico-pedagógico-didáctico del proceso de la formación de los docentes y registran posibilidades de desarrollo de una enseñanza de las ciencias con sus métodos científicos, pero no el centro de la formación de los docentes del país: lo filosóficopedagógico-didáctico-psicológico-sociológico necesarios.

\section{Gestión Curricular}

Es indiscutible que la gestión adecuada del currículo busca el éxito del modelo educativo, los planes de estudio y las formas de evaluación de los aprendizajes de los estudiantes, pues se apuesta por una sistematización y diseño de acciones didácticas para la mejora, específicamente en la organización y desarrollo de la enseñanza, el aprendizaje y la evaluación curricular. Tareas que ejercen los decanos y jefes de departamentos o secciones académicas, quienes son los encargados de apoyar la labor docente en el aula y, por ende, de incentivar el avance de la formación docente de un profesor en la institución.

La realización de estas tareas implica el desarrollo de un trabajo planificado y coordinado por la Dirección de Desarrollo Curricular centrada en tres acciones: La constitución de redes de acompañamiento docente; el fortalecimiento del guión metodológico; y, la formación docente como el objetivo central de los profesores universitarios.

Las redes de acompañamiento docente potencian la convivencia y solución a problemas que se activan al unir esfuerzos para sistematizar la experiencia docente, y son integradas por grupos de docentes, sin jerarquías, para intercambiar información y coordinar actividades inspiradas en la metodología de la investigación acción. El guión metodológico es la herramienta básica de la planificación didáctica de los profesores que sirve de apoyo en la labor docente e incluye orientaciones que susciten el aprendizaje, la enseñanza y la evaluación de las competencias en cada espacio pedagógico. 
La formación docente se sustenta en las Normas Académicas de Educación Superior (art. 157) relacionadas con la evaluación y la universidad define algunos factores e indicadores que ayudarán al desarrollo de acciones sistemáticas de monitoreo y evaluación en el proyecto de aula, centradas en el guión metodológico, el portafolio del docente, el portafolio del estudiante, para evidenciar los alcances académicos obtenidos.

Como se observa, el modelo educativo de la UPNFM tiene vacíos teórico-metodológicos respecto de la formación docente basado en competencias, de los conocimientos metodológicos con los que los estudiantes deben egresar, de un sistema de evaluación de los aprendizajes por lo que parece conveniente acercar ciertas dimensiones teóricas, epistemológicas (Gorodokin, 2005) y metodológicas que orienten a la formación docente de un profesor competente (Tobón, 2015). A continuación, se presentan estos agregados:

\section{La Formación Docente de un Profesor}

El enfoque de la formación basado en competencias sugerido por Tobón (2015) implica el desarrollo de los saberes: ser, conocer y hacer, en ese orden. Esta perspectiva de la formación docente implica avanzar al discurso de Barnett (2001) sobre las competencias, pues para este último autor, en la formación en competencias, se debe superar lo operacional de ellas para ir a lo académico, que es el dominio de las ciencias, pero no quedarse allí, pues es necesario asegurar que el profesor formado alcance las competencias del mundo de la vida en las aulas y de esa manera asegurar la formación de profesores competentes, no competitivos.

Esta visión, supone la presencia de un profesor que en todo acto académico muestre una actitud científica de respaldo. Siguiendo a Bourdieu (2002):

Un campo -podría tratarse del campo científico- se define, entre otras formas, definiendo aquello que está en juego y los intereses específicos, que son irreductibles a lo que se encuentra en juego en otros campos o a sus intereses propios (...). Para que funcione un campo, es necesario que haya algo en juego y gente dispuesta a jugar, que esté: dotada de los habitus que implican el conocimiento y reconocimiento de las leyes inmanentes al juego, de lo que está en juego, etcétera. (p. 120) 
El campo científico y el habitus es un sistema de relaciones objetivas entre posiciones adquiridas donde se lucha por el monopolio de la autoridad científica, que da capacidad técnica y poder social y el que permite la conformación de un discurso pedagógico sólido en la enseñanza y el aprendizaje.

Esto pondría al profesor en capacidad de ir más allá de la enseñanza como práctica, que implica avanzar a la enseñanza como conocimiento o, mejor aún, a la enseñanza como posibilidad de pensamiento (Martínez B., 2003) que encuadra con lo que Carr (1999) dice respecto de las prácticas pedagógicas en las que se deben superar las prácticas de las prácticas para avanzar en la aplicación de al menos una teoría de las prácticas (la práctica se opone a la teoría, la práctica depende de la teoría o que existe una autonomía en las prácticas) o, más allá, desarrollar una verdadera integración de las teorías con las prácticas (Umanzor, 2011).

La conjunción de las lógicas de la enseñanza pasa por el entronque y derivaciones de los conocimientos metodológicos asociados a un método, a técnicas, a estrategias y a procesos de enseñanza que le dan sentido al proceso de enseñanza-aprendizaje, mismo que procuraría la formación docente de un profesor competente.

En esta dirección, se considera lo dicho por Nérici (1985) quien dijo que: "Didácticamente, método quiere decir camino para alcanzar los objetivos estipulados en un plan de enseñanza". El punto de discusión es cómo se cómo se logran los objetivos de ese camino. ¿Cuáles son los momentos de reflexión que deben desarrollarse para definir ese camino? ¿Qué técnicas de enseñanza son las apropiadas para avanzar en ese camino? ¿Qué estrategias de enseñanza son las recomendadas por el método para avanzar en ese camino? Y ¿qué procesos de enseñanza favorecen cada momento de los aprendizajes de los estudiantes?

El mismo autor sugiere que una técnica de enseñanza "significa cómo hacer algo". Entonces, ese hacer algo atraviesa por diferentes formas de actuaciones que debe seguir un profesor para asegurar que los estudiantes aprendan mediante dinámicas que deben jugar los grupos de estudiantes en el aula porque en el avance del camino hay un método y también técnicas apropiadas para llegar al fin del camino trazado. 
En ese avance hay que llegar al fin del camino y por eso se deben utilizar, también, estrategias de enseñanza que fortalezcan la creatividad de los profesores y el aprendizaje de sus estudiantes, por eso Vera y Vera G. (2011) sugieren que estrategias son "los diversos momentos o fases de la clase a fin de lograr la eficacia en el proceso de enseñanzaaprendizaje, donde se evidencie la participación del estudiante" (pp. 399-400). Una pregunta problematizadora oportuna, la presentación de un vídeo, una lámina donde se pueda analizar, sintetizar y evaluar su contenido contribuiría en esa búsqueda del fin del camino.

También, en ese camino son necesarios los procesos inductivos o deductivos para fortalecer los aprendizajes de los estudiantes y como Alfonso Sánchez (2003) dice que: "El proceso de enseñanza, con todos sus componentes asociados, debe considerarse como un sistema estrechamente vinculado con la actividad práctica del hombre, que, en definitiva, condiciona sus posibilidades de conocer, comprender y transformar la realidad que lo circunda". (penúltimo párrafo, apartado La enseñanza)

Significa que estos procesos son los que direccionan y perfeccionan constantemente el quehacer cognoscitivo del hombre que deberá desarrollarse hasta alcanzar el fin del camino. Esto implica ir de lo particular a lo general o de lo general a lo particular y de esa manera encontrar el patrón que evidencie el aprendizaje de los estudiantes. No son caminos opuestos, son complementarios, pues el resultado final debe ser que el estudiante alcance un razonamiento lógico para su desarrollo como profesional.

En tal caso, el método indica el camino, la técnica muestra cómo recorrerlo, las estrategias (procedimientos) cómo profundizar el desarrollo de los contenidos curriculares y los procesos cómo organizar el pensamiento lógico de los estudiantes. Es de esta manera que se construyen los conocimientos metodológicos que en esta investigación se validan.

Por tanto, los conocimientos metodológicos como dice Nérici (1985) “son un conjunto de procedimientos didácticos, implicados en los métodos y técnicas de enseñanza [así como las estrategias y procesos de enseñanza], que tienen por objeto llevar a buen término la acción didáctica, es decir, alcanzar los objetivos de la enseñanza y, en consecuencia, los 
de la educación, con un mínimo de esfuerzo y un máximo de rendimiento” (pp. 35-36).

En resumen, los conocimientos metodológicos están asociados al manejo estricto de las concepciones sobre la enseñanza y las prácticas pedagógicas contextualizadas que satisfacen la formación de profesionales aptos para el desempeño de una actividad científica, empírica o práctica en función de las acciones didácticas desarrolladas en las aulas.

Como la didáctica es una ciencia, entonces, sus requerimientos son sustentaciones de unas prácticas pedagógicas orientadas con los conocimientos metodológicos propios de esa ciencia, para observar, experimentar, imaginar, intuir y, con ello, desarrollar el pensamiento lógico de los estudiantes de manera inductiva o deductiva.

Estos conocimientos metodológicos deben responder en cualquier momento y circunstancia a sus comprensiones y aplicaciones hasta lograr una manera en la que los profesores sean competentes, que como dice Marcelo (comunicación personal, 25 de junio del 2020) la formación docente de un profesor es un:

proceso mediante el cual las personas en cualquier momento de su vida (en formación, en ejercicio, sin actividad, jubilados, etc.) se implican individualmente o en grupo, en experiencias de aprendizaje a través de las cuales adquieren o mejoran competencias, que les permiten su desarrollo personal y profesional y el de la organización para la cual trabajan.

De allí que la formación docente de un profesor pasa por la actitud científica que adopten los formadores de formadores en la enseñanza de las ciencias y en la formación de estudiantes-profesores que sepan la ciencia a enseñar, pero fundamentalmente se apropien de los conocimientos filosóficos, pedagógicos, didácticos, psicológicos y sociológicos para ejercer la docencia como profesionales que saben enseñar desde el aprender.

\section{Métodos y Materiales}

Para realizar esta investigación se asumió la metodología de la investigación cuantitativa con diseño no experimental porque se trata de describir y explicar cómo las percepciones de los estudiantes sobre sus conocimientos metodológicos mediante una Regresión 
Lineal Múltiple a partir de una Escala Likert. Este es un instrumento que mide, como dice Sierra Bravo (2003), “aspectos de una característica o actitud, a los que se asigna convencional o empíricamente un valor numérico, lo que permite cuantificar el grado de esa característica o actitud que se da en un objeto o sujeto determinado". (p. 370)

Es una escala de actitud con base ordinal, inicia con valores de 1 a 5 y desde allí se construyó el objeto de la validación de las percepciones de los estudiantes sobre sus conocimientos metodológicos haciendo los ajustes estadísticos necesarios y suficientes que apoyaron esa validación. Siguiendo a Suárez Parra (2014):

En la validación de escalas, la Estadística cobra importancia en la fase cuantitativa de este proceso, ya que es la ciencia que respal $\neg$ da los resultados numéricos para la toma de decisiones. Con esta intensión se presentan las técnicas estadísticas de mayor uso en la validación de escalas tipo Likert agrupadas en dos componentes: validez interna y validez externa. (p. 47)

Con esta escala se espera establecer la magnitud de cambio que pudiera experimentar en el tiempo la validez interna de la escala o con el criterio de expertos como validez de constructo o mediante la prueba de confiabilidad de Alpha Cronbach. Esto sugiere la capacidad de determinación de los ítems de la escala y garantiza el camino hacia la objetivación de este tipo de validez, pues la exigencia cuantitativa en la validación de escalas se da calculando correlaciones de Pearson o Regresión Lineal Múltiple que son las “más usuales para evidenciar validez externa” (p. 58) considerando que los resultados aceptables son cuando " $\mathrm{r} \geq .25$ entre las mismas variables". (p. 51)

Este es un estudio transeccional porque se hizo una sola medición de las variables con los estudiantes de los espacios pedagógicos: Metodología de Investigación Cuantitativa y Metodología de Investigación Cualitativa con 91 estudiantes, matriculados en el II período académico del 2020, de los cuales 76 pertenecían al primer espacio pedagógico (varias carreras) y 15 estudiantes de matemáticas al segundo espacio pedagógico. Es una muestra por conveniencia porque los estudiantes estaban inscritos en esos espacios 
pedagógicos. La representatividad de la población de estudiantes del CURSPS-UPNFM, en esta muestra, está garantizada porque se logra la normalidad con 50 estudiantes.

La escala Likert se elaboró con 57 reactivos (afirmaciones) divididos en las variables: modelo educativo (7), planes de estudios (9), guión metodológico (4), planes de clases (6), conocimientos metodológicos (17), así como recursos y medios (7) y formas de evaluación de los aprendizajes (6). Las valoraciones iniciales de la escala fueron: 1. No conozco, 2. Conozco un poco, 3. Conozco suficiente, 4. Conozco bastante, y, 5. Conozco completamente.

Para validar esta escala, primero se hizo una prueba piloto con cinco expertos: dos especialistas en matemáticas, uno en ciencias naturales y del medio ambiente, uno en lenguas-español y otro en lenguas-inglés. Sus sugerencias fueron incorporadas en la escala y con ello se fortalecieron los alcances de las variables y sus afirmaciones, y así se consolidó el instrumento. Mismo que se trasladó a Formularios de Google y se envió su enlace por WhatsApp a los correos electrónicos de cada estudiante, previo consentimiento en una videoconferencia.

Para la construcción de las variables cuantitativas y de nuevo a ordinales se utilizó el software SPSS, v. 25, acompañado de otro llamado GPower, v. 3.1 para generalizar los resultados a la población y eso implicó realizar las pruebas: de confiabilidad, de normalidad, de homocedasticidad para cumplir con los requisitos de las correlaciones de Pearson a las que se agregaron sus efectos y potencias entre las variables del estudio. Siguiendo a Manzano-Arrondo (2009) el tamaño del efecto es un concepto que sirve para calcular la potencia de una prueba y a partir de ellas interpretar el valor encontrado. El tamaño del efecto máximo es B $>0.5$ y se aplica tanto a correlaciones de Pearson y Spearman como a la Regresión Lineal Múltiple.

También, para fortalecer la validación de las percepciones de los estudiantes sobre sus conocimientos metodológicos se agregó la prueba Durbin-Watson (1951) para explicar la independencia de las relaciones de las variables desde los residuos en la Regresión 
Lineal Múltiple, que como lo dice Moreno González (2008) su valor oscila entre 0 y 4, pero es sustentable si alcanza valores entre 1.5 y 2.5 .

Estos criterios le dan el sentido estadístico cuantitativo que requieren los constructos en este proceso de validación de las percepciones de los estudiantes sobre sus conocimientos metodológicos (variable dependiente) en función de variables independientes: modelo educativo, planes de estudio, guión metodológico, planes de clases, recursos y medios, y formas de evaluación de sus aprendizajes.

\section{Resultados}

Este estudio muestra la situación sociodemográfica, laboral y de conectividad a internet de los estudiantes respecto de su formación como profesores; se construyeron las variables necesarias para establecer las dimensiones paramétricas que ayudaron a culminar con la validación de los conocimientos metodológicos predichos. A continuación, se presentan los resultados descriptivos e inferenciales básicos del estudio:

\section{Análisis Descriptivo}

Los resultados muestran que los estudiantes consultados en un 34\% son varones y el $66 \%$, mujeres. Con una edad promedio de 25 años y una variación de 6 años; donde el $25 \%$ es menor de 21 años, el 50\%, menor de 23 años y el 75\%, menor de 27 años. Son estudiantes con edad que oscila entre 19 y 31 años, edad ideal para su formación como docentes, considerando que el $85 \%$ trabaja y el 77\% lo hace como profesor. Ello implica que su proceso de formación sea lento y más si solamente matriculan un promedio de 3 espacios pedagógicos por período, aunque oscila entre 2 y 4.

Eso tiene un agravante, estudian apenas, en promedio, 3 horas diarias con una variación de 1.5 lo que confirma que sus horas de estudio se dan entre 1.5 y 4.5 en conjunto los 3 espacios pedagógicos matriculados; mucho menor de lo estipulado para estudiar en casa, un mínimo de 2 horas de estudio diario por hora-clase recibida, considerando que un $76 \%$ de ellos aún son solteros y con una media de 0.30 hijos. 
Del $77 \%$ de los estudiantes que trabajan como docentes, el 14\% lo hace en educación prebásica, el $59 \%$ en básica y el $27 \%$ en media. Esta experiencia docente acumulada debería ser insumo para una mejor formación como docente, para fortalecer sus prácticas pedagógicas o fomentar la formación como docentes de los otros estudiantes, pues el 46\% sean bachilleres en ciencias y letras, el 23\% bachilleres técnicos y el $31 \%$ maestros de educación primaria y dedican jornadas, en promedio, de 8 horas diarias. El 27\% de estos enseña inglés y el $45 \%$ enseña varias asignaturas a la vez en varios grados. El resto lo hace en un abanico de cursos.

Muchos de ellos trabajaban, antes de la pandemia, en la modalidad de enseñanza presencial (41\%), pero ahora, todos lo hacen en una combinación virtual-a distancia. Para ello utilizan o solo plataformas (32\%), o WhatsApp-plataformas (27\%), o sólo WhatsApp (23\%) y otros sólo vídeos (18\%). Esto significa que hacen uso de las tecnologías de la información y la comunicación, ventaja que debe aprovechar la UPNFM para incorporar la virtualidad en la formación de docentes, a pesar de que su conectividad en su celular sea preferentemente de 1-5 Gb (59\%) y de 6-10 Gb (16\%), y el resto combina más altos niveles de conectividad; $\mathrm{y}$, en su casa, los niveles de conectividad son parecidos, de 1-5 Mb (59\%) y de 6-10 Mb (16\%), y un resto que combina niveles más altos.

Luego, en el afán de mayores descripciones las variables cuantitativas se transformaron en variables ordinales, para definir nuevos niveles, así: 1 . No tiene conocimientos, 2 . Tiene conocimientos básicos, y, 3. Tiene conocimiento total sobre las variables asociadas; se determinaron los intervalos de confianza $[\mu \pm \sigma(.75)]$ que facilitaron estos niveles. Con ello se logró posicionar sus percepciones en los conocimientos básicos (segundo cuartil), con porcentajes que oscilan entre $17 \%$ y $24 \%$ en el primer nivel; en el segundo estos porcentajes van desde $50 \%$ a $59 \%$; y, en el tercero entre el $18 \%$ y $26 \%$.

Todos los estudiantes consultados tienen computadora y teléfono inteligente, pero una buena parte recibe las clases comprando internet para el momento; más de la mitad tiene una conectividad baja en su casa y en su celular. Con las experiencias docentes adquiridas por estos estudiantes, a pesar de los problemas de conectividad, habría condiciones 
teórico-metodológico-tecnológicas para la formación docente de un profesor en el marco de la virtualidad.

Como el interés de esta investigación es validar los conocimientos metodológicos de los estudiantes en la propuesta del modelo educativo y sus componentes se aplicaron las pruebas correspondientes y se encontró una confiabilidad $\alpha=0.98$, una normalidad de Kolmogorov-Smirnov $=0.20>0.05$. Y una prueba de Levene (igualdad de varianzas $)=0.33$ $>0.05$, lo que sugiere que existe homocedasticidad, por tanto, se cumple con los requisitos para la validación de las percepciones de los estudiantes respecto de sus conocimientos metodológicos. Luego, para garantizar la validación de la escala, se calculó la correlación de Pearson (r) y los resultados son los que se muestran en la Tabla 1:

\section{Análisis Inferencial}

Análisis de Correlación de Pearson (r)

Tabla 1

Relación de las percepciones de los estudiantes sobre sus conocimientos metodológicos con las de las otras variables

\begin{tabular}{lccccc}
\hline $\begin{array}{c}\text { Conocimientos metodológicos de los } \\
\text { estudiantes y }\end{array}$ & $\mathrm{n}$ & $\begin{array}{c}\text { Pearson } \\
(\mathrm{r}= \pm 1)\end{array}$ & $\begin{array}{c}\text { Sig } \\
(\alpha=.05)\end{array}$ & $\begin{array}{c}\text { Efecto } \\
(\mathrm{f}=.5)\end{array}$ & $\begin{array}{c}\text { Potencia } \\
(1-\beta=.8)\end{array}$ \\
\hline Modelo educativo & 91 & $.82^{* *}$ & 0.001 & 0.91 & 1 \\
Planes de estudio & 91 & $.74^{* *}$ & 0.001 & 0.86 & 1 \\
Guión metodológico & 91 & $.79 * *$ & 0.001 & 0.89 & 1 \\
Plan de clases & 91 & $.85^{* *}$ & 0.001 & 0.92 & 1 \\
Recursos y medios & 91 & $.79^{* *}$ & 0.001 & 0.89 & 1 \\
Formas de evaluación aprendizajes & 91 & $.78^{* *}$ & 0.001 & 0.88 & 1 \\
\hline
\end{tabular}

Nota: Los asteriscos dobles indican que las correlaciones de Pearson son significativas al $\alpha=$ 0.05 y al $\alpha=0.01$.

Como se puede observar en la Tabla 1, las variables indicadas están correlacionadas positiva y de manera alta entre los conocimientos metodológicos y los de las otras variables, son significativas al $\alpha=0.05$ y al $\alpha=0.01$, y oscilan entre .74 y .85. Con un efecto entre el 
$86 \%$ y el 92\% y una potencia del 100\%, respectivamente, en cada relación. Esto supera lo planteado por Suárez Parra y con ello se determina la validez de constructo, la interna y la externa de la Escala Likert.

Considerando estos resultados, ahora se procedió a predecir la influencia que reciben los conocimientos metodológicos de los alcances del modelo educativo, de los planes de estudio, del guión metodológico, de los planes de clases, de los recursos y medios, y de las formas de evaluación de los aprendizajes. Para lograrlo, se aplicó el estadístico Regresión Lineal Múltiple (método escalonado), para determinar los factores que explican las variaciones de los conocimientos metodológicos y se muestran en la Tabla 2, a continuación:

\section{Análisis de regresión múltiple (método escalonado)}

Para explicar los alcances de las percepciones de los conocimientos metodológicos se agregó otra prueba llamada Durbin-Watson (1951) y el resultado de esta prueba fue 2.094, para indicar que, en conjunto, planes de clase, recursos y medios, planes de estudio y las formas de evaluación predicen el 85\% de los conocimientos metodológicos de los estudiantes.

Tabla 2

Predicción de los conocimientos metodológicos de los estudiantes según modelos

\begin{tabular}{lccccccccc}
\hline \multicolumn{1}{c}{ Modelos } & F & R2 & AR2 & B & $\begin{array}{c}\text { Error } \\
\text { estándar }\end{array}$ & $\beta$ & p & f2 & $1-\beta$ \\
\hline Modelo 1 & 225.69 & 0.72 & 0.71 & 2.435 & 0.162 & 0.847 & 0.001 & 0.85 & 1 \\
Conocimiento plan de clases & $(1,89)$ & & & & & & & & \\
Modelo 2 & 177.049 & 0.8 & 0.8 & & & & 0.001 & 0.89 & 1 \\
Conocimiento plan de clases & $(2,88)$ & & & 1.665 & 0.186 & 0.579 & 0.001 & & \\
Conocimiento recursos y medios & & & & 0.965 & 0.158 & 0.394 & 0.001 & & \\
Modelo 3 & 141.27 & 0.83 & 0.82 & & & & 0.001 & 0.91 & 1 \\
Conocimiento plan de clases & $(3,87)$ & & & 1.284 & 0.2 & 0.446 & 0.001 & & \\
Conocimiento recursos y medios & & & & 0.852 & 0.15 & 0.349 & 0.001 & & \\
Conocimiento planes de estudio & & & & 0.498 & 0.13 & 0.238 & 0.001 & \\
Modelo 4 & 119.507 & 0.85 & 0.84 & & & & 0.001 & 0.92 & 1 \\
Conocimiento plan de clases & $(4,86)$ & & & 1.046 & 0.204 & 0.364 & 0.001 & \\
Conocimiento recursos y medios & & & & 0.518 & 0.178 & 0.212 & 0.005 & \\
Conocimiento planes de estudio & & & & 0.534 & 0.124 & 0.255 & 0.001 & \\
Conocimiento formas de evaluación & & & & 0.625 & 0.197 & 0.233 & 0.002 & \\
\hline
\end{tabular}


Con los resultados obtenidos en la Tabla 2, se observan 4 escenarios: con el primero, los conocimientos sobre el plan de clases bastan para explicar el 72\% los conocimientos metodológicos considerando un $\alpha=0.001$, un ANOVA $(\mathrm{F}=225.690, \mathrm{gl}=1,89)$ y un error estándar muy bajo; eso determina un efecto de .85 y una potencia del $100 \%$, lo que sugiere que, con solo el conocimiento, uso y manejo adecuado del plan de clases estaría garantizada la formación docente de un profesor. Basta con que el profesor planifique sus clases con la idea generar procesos de análisis crítico, reflexivo y creativo, para formar un docente.

Un segundo escenario sugiere que, además de tener claros sus conocimientos sobre los alcances positivos del plan de clases, el profesor debe asegurarse que los recursos y medios con los que desarrolle su enseñanza sean los que necesita para garantizar la construcción de los materiales didácticos necesarios y suficientes, ahora es indispensable el conocimiento, uso y manejo adecuado de los tecnológicos para la virtualidad en la formación docente de un profesor. Los resultados se incrementaron a un $80 \%$ con un $\alpha=0.001$, un ANOVA (F $=$ $177.049, \mathrm{gl}=2,88)$ y un error estándar muy bajo, con un efecto de .89 y una potencia del $100 \%$. Significa que se puede mejorar la formación docente de un profesor con el manejo del plan de clases en la enseñanza y el uso y manejo adecuados de los recursos y medios, especialmente los tecnológicos para la formación docente en una pandemia sanitaria.

Un tercer escenario sugiere que, además de que los profesores tengan un claro conocimiento, uso y manejo de los de los planes de clases y de los recursos y medios, especialmente los tecnológicos, sus conocimientos metodológicos se mejorarán con su conocimiento sobre los planes de estudio, pues con ello sabrán para qué ser profesor, qué se debe aprender y cómo aprender ser profesor. Los resultados sugieren que sus competencias se incrementaron a un $83 \%$ con un efecto de .91 y una potencia del $100 \%$, a partir de un $\alpha=0.001$, un ANOVA (F $=141.270, \mathrm{gl}=3,87)$ y un error estándar bajo.

Y el cuarto escenario sugiere que, bajo las características del tercer modelo, todos los conocimientos metodológicos de los estudiantes, más el conocimiento sobre las formas de evaluación de sus aprendizajes, se incrementaron a un $85 \%$ con un efecto de .92 y una potencia del $100 \%$, con un $\alpha=0.001$, un ANOVA $(\mathrm{F}=119.507, \mathrm{gl}=4,86)$ y un error estándar bajo. 
Significa que la formación docente de un profesor pasa porque los estudiantes tengan claros sus conocimientos metodológicos, mismos que son explicados por el conocimiento que tengan sobre los alcances y bondades del uso y manejo adecuados del plan de clases en la enseñanza, del papel de los recursos y medios en la construcción de los materiales didácticos apropiados, del sentido de pertinencia, eficiencia y eficacia de los planes de estudio para una correcta formación docente, y de las formas de evaluación justas de sus aprendizajes.

El modelo de regresión lineal múltiple (método escalonado) eliminó del análisis las variables modelo educativo y guión metodológico, lo que indica que conocer sus alcances tiene implicaciones más administrativas que académicas, por tanto, en las circunstancias actuales, las percepciones de los conocimientos metodológicos de los estudiantes dependen más del desarrollo sistemático de los conocimientos de los planes de clase, de los recursos y medios, de los planes de estudio y de las formas de evaluación de los aprendizajes adquiridos en su proceso de formación como docentes.

\section{Consideraciones Finales}

El propósito de la investigación era validar la percepción de los conocimientos metodológicos de los estudiantes en función de los del modelo educativo y sus componentes, y se encontró que:

1. Los datos obtenidos muestran a una población de estudiantes del CURSPS-UPNFM con debilidades económicas, académicas, laborales y de conectividad para el desarrollo de una educación virtual a plenitud, pues, aunque muchos estudiantes trabajan como docentes, viven circunstancias carenciadas con el conocimiento, uso y manejo adecuados de los recursos tecnológicos para ser formados como profesores.

Lamentablemente, la pandemia obligó a sus docentes, también, a aprender, por su cuenta, en principio, a conocer, usar y manejar esos recursos tecnológicos y eso debilita su formación como docentes. No hay duda de que las competencias docentes se están construyendo en la práctica de las prácticas, especialmente por lo apretado con que se producen los conocimientos de la tecnología, pero los costos de esos aprendizajes, por mucho, son sufragados por los mismos docentes porque su trabajo está en cuestión y se debe avanzar a la construcción de respuestas educativas sobre la marcha. 
2. Los resultados indican que los estudiantes muestran niveles básicos en sus conocimientos metodológicos, así como sobre el modelo educativo, planes de estudio, guión metodológico, recursos y medios, y las formas de evaluación de sus aprendizajes.

Esto significa que sus percepciones actuales sobre las bondades del modelo educativo y sus componentes pueden estar determinadas por la falta de acercamientos a la ideología y filosofía de la universidad en los espacios pedagógicos que sirven los docentes en sus cátedras.

En estas circunstancias conviene evaluar qué de lo declarado en el modelo educativo y sus componentes tiene efectos positivos en el cambio de concepciones de los estudiantes sobre la enseñanza y el desarrollo de las prácticas pedagógicas que facilitan su formación como docentes.

3. Los resultados indican que las correlaciones entre las variables en estudio son altas y muy altas, todas positivas y altamente significativas, por tanto, están por encima de las normas que se exige de correlaciones Pearson para validar escalas.

Esto es garantía de que la validación de la escala tuvo el efecto esperado, por tanto, la búsqueda, ahora, debe ser el fortalecer los conocimientos de estudiantes sobre el modelo educativo y sus componentes de manera de fortalecer sus visiones sobre el ser profesor, no un especialista.

La formación docente de los estudiantes universitarios está imbricada en la construcción de conocimientos científicos y fundamentalmente pedagógicos para asegurar una docencia eficiente y eficaz. El ser un profesor competente condiciona a formadores de formadores y a los estudiantes-profesores a aprender a enseñar para enseñar a aprender desde el aprender.

Con el modelo de regresión lineal múltiple (método escalonado) se pudo establecer que los factores que explican las variaciones de los conocimientos metodológicos de los estudiantes universitarios son sus saberes sobre los planes de clases, los recursos y medios, los planes de estudio y las formas de evaluación de sus aprendizajes, en este orden, con un alto grado estadístico.

Esto significa que es vital la construcción de un modelo de plan de clases en la enseñanza para fortalecer las competencias docentes de un profesor que debe saber 
enseñar; de prototipos de equipos educativos y de materiales didácticos afines a las cátedras, de un sistema de capacitación a los profesores para la adopción de las tecnologías apropiadas, así como de elaboración del material didáctico, incluyendo los textos con los que cada profesor enseña.

Fundamentalmente, es el momento de revisar los planes de estudio para garantizarle a los estudiantes un aprendizaje sistemático sobre las ciencias con sus métodos científicos, en el nivel que corresponda, pero, preferentemente, en la construcción de una filosofía de enseñanza propia del ser profesor, en la determinación de una pedagogía formadora de un profesor, en la elaboración de los planes de clases que la universidad considera válidos; y en esa línea con los conocimientos metodológicos amplios para que cada docente decida qué método de enseñanza utilizar en qué circunstancia (método diálogo reflexivo, Umanzor, 2011), qué técnicas satisfacen las exigencias de ese método; qué estrategias fortalecen el desarrollo de la enseñanza crítica, reflexiva y crítica en apoyo a un método; y, sobre todo qué proceso de enseñanza conviene utilizar en cada momento de aprendizaje de los estudiantes hasta fortalecer sus razonamientos lógicos y de esa manera alcanzar su formación como profesores críticos, reflexivos y creativos como lo plantea el modelo educativo.

También, saber diferenciar qué teoría de aprendizaje conviene para qué casos, y cómo fortalecer las competencias psicológicas de los docentes para orientar el mejoramiento del rendimiento académico de sus estudiantes. Y, finalmente, cómo dirigir procesos de enseñanza donde los conflictos y los maltratos psicológicos (bullyings) son la norma en los comportamientos de los estudiantes de los niveles a los que estos profesores accederán y de esa manera garantizar una formación docente competente.

Asimismo, habría una mejor respuesta de los estudiantes para ser formados como docentes sabiendo que sus evaluaciones son justas. Que los tipos de evaluaciones: diagnósticas, formativas y sumativas responden a un sistema en el que ellos son parte activa y donde existen claras evidencias de la valoración de sus aprendizajes.

Se asume, entonces, que un docente sabrá enseñar desde el aprender cuando la universidad los forme para esa profesión y el camino empieza en la revisión del modelo educativo y sus componentes en la perspectiva de Imbernón (2007): formación inicial, permanente y profesional de los docentes. 


\section{Referencias}

Alfonso Sánchez, I. (2003). Elementos conceptuales básicos del proceso de enseñanzaaprendizaje. $A C I M E D, 11(6)$. http://scielo.sld.cu/scielo.php?script=sci_arttext\&pid $=\mathrm{S} 1024-94352003000600018$

Barnett, R. (2001). Los límites de las competencias: el conocimiento, la educación superior y la sociedad. Gedisa.

Bolívar, A. (2005). Conocimiento Didáctico del Contenido y Didácticas Específicas. Currículum y formación del profesorado, 9, 2 (2005). https://www.ugr.es/ recfpro/ rev92ART6.pdf

Bourdieu, P. (2002). Campo de poder, Campo intelectual. Itinerario de un concepto. Montressor. http://ceiphistorica.com/wp-content/uploads/2016/01/bourdieu-campode-poder-campo-intelectual.pdf

Carr, W (1999). Una teoría para la educación. Hacia una investigación educativa crítica. Morata.

Diaz Barriga, A. (1986). La formación del pedagogo. Un acercamiento al tratamiento de los temas didácticos en el plan de estudios. Conferencia-UNAM. http://publicaciones. anuies.mx/pdfs/revista/Revista64_S2A1ES.pdf

Díaz Barriga, A. (2005). El docente y los programas escolares: Lo institucional y lo didáctico. Pomares. https:/www.dgespe.sep.gob.mx/public/gt-en/acuerdos/subcomisiones/ RC/17-19Ene2011/material/El\%20Docente\%20y\%20los\%20Programas\%20Escolares. $\mathrm{pdf}$

Gallego, R. y Pérez, R. (1998). Aprendibilidad, enseñabilidad y educabilidad en las ciencias experimentales. Educación y Pedagogía, XI(25), 87-117. https://scholar.google.es/ scholar?cluster $=6571719960950589070 \& h l=$ es\&as_sdt $=0,5$

Gorodokin, I. C. (2005). La formación docente y su relación con la epistemología. Iberoamericana de Educación, 37(5, extra), 1-9. https://dialnet.unirioja.es/servlet/ articulo? codigo $=3197461$

Imbernón, F. (2007). La Formación y el desarrollo profesional del profesorado. Hacia una nueva cultura profesional. Graó. 
Lorenzo, M. G. (2017). Enseñar y aprender ciencias. Nuevos escenarios para la interacción entre docentes y estudiantes. Educación y Educadores, 20(2), 249-263. https://doi: 10.5294/edu.2017.20.2.5

Manzano-Arrondo, V. (2009). Sobre la acotación de efectos y la existencia de relación. http://asignatura.us.es/dadpsico/apuntes/efectos.pdf

Marcelo, C. (2020). La formación docente de un profesor. https://www.researchgate.net/ profile/Carlos_Marcelo.

Martínez B., A. y los miembros de la Revista Pedagogía y Epistemología. (2003). La enseñanza como posibilidad de pensamiento. Magisterio. http://www.albertomartinezboom.com/ escritos/libros/2003_Pedagogia_y_epistemologia.pdf

Moreno González, E. (2008). Manual de Uso de SPSS. Guías Prácticas (1 $1^{\mathrm{a}}$ ed.). UNED. http://e-spacio.uned.es/fez/eserv/bibliuned:500727/Guia_SPSS.pdf

Nérici, I. G. (1985). Metodología de la Enseñanza. Kapelusz Mexicana.

Rodríguez Rivera, D. E., López Molina, E. J., Arias Galindo, H. E., Carlos Alfredo Mejía González, C. A. y García Menjívar, R. A. (2020, primer período). El compromiso de los estudiantes favorece el aprender y enseñar matemáticas en competencias (Manuscrito no publicado), Sección Académica: Ciencias Matemáticas, espacio pedagógico: Seminario de Investigación II, CURSPS-UPNFM.

Sierra Bravo, R. (2003). Técnicas de Investigación Social. Teoría y Ejercicios (Decimocuarta ed.). Thomson.

Suárez Parra, A. B. (2015). La estadística en la validación de escalas, una visión práctica para su construcción o su adaptación. Revista I3+, 2(2), 46-61. https://doi.org $/ 10.24267 / 23462329.111$

Tobón, S. (2015). Formación basada en competencias. Pensamiento complejo, diseño curricular y didáctica. https://www.uv.mx/psicologia/files/2015/07/Tobon-S.Formacion-basada-en-competencias.pdf

Umanzor, P. (2011). Construcción de un diálogo reflexivo a partir de prácticas pedagógicas y concepciones sobre la enseñanza. (Tesis de Doctorado). UPNFM, Repositorio. 
Umanzor, P. (2018). Epistemología de la formación docente: una mirada crítica. Paradigma, 23(36):35. https://doi: 10.5377/paradigma.v23i36.6486

Umanzor, P. y Castro, D. A. (2019). Formación de un Profesor Investigador en una Comunidad de Aprendizaje. UNAH-VS, CRIC, Aportando Conocimiento, 1(1), 26-34. UPNFM (2014). Modelo Educativo. Universitaria.

Vera, A. C. y Vera G., L. J. (2011). Estrategias utilizadas por los docentes para promover el aprendizaje de la biología a nivel universitario. Telos, 13(3), 397-411. https://www. redalyc.org/pdf/993/99320590008.pdf

Zabalza, M. A. (2009). Ser profesor universitario hoy. La Cuestión Universitaria, 5(2009), 68-80. http://polired.upm.es/index.php/lacuestionuniversitaria/article/view/3338/3403 
102 <Paradigma> Revista de Investigación Educativa | Año 27 | N44 | Julio-Diciembre 2020 | ISSN: 1817-4221 | EISSN: 2664-5033

(c) (†) Los artículos de la Revista Electrónica Paradigma del Instituto de Investigación y Evaluación Educativas y Sociales de la Universidad Pedagógica Nacional cC $\mathrm{EY}$ No ND Francisco Morazán, Honduras, se comparten bajo términos de la Licencia Creative Commons: Reconocimiento, No Comercial, Sin Obra Derivada 